\title{
Adhesive rough contacts near complete contact
}

\author{
M. Ciavarella \\ Politecnico di BARI. Center of Excellence in Computational Mechanics. Viale Gentile \\ 182,70126 Bari.Mciava@poliba.it
}

\begin{abstract}
Recently, there has been some debate over the effect of adhesion on the contact of rough surfaces. Classical asperity theories predict, in agreement with experimental observations, that adhesion is always destroyed by roughness except if the amplitude of the same is extremely small, and the materials are particularly soft. This happens for all fractal dimensions. However, these theories are limited due to the geometrical simplification, which may be particularly strong in conditions near full contact. We introduce a simple model for adhesion, which aims at being rigorous near full contact, where we postulate there are only small isolated gaps between the two bodies. The gaps can be considered as "pressurized cracks" by using Ken Johnson's idea of searching a corrective solution to the full contact solution. The solution is an extension of the adhesive-less solution proposed recently by $\mathrm{Xu}$, Jackson, and Marghitu (XJM model) (2014). This process seems to confirm recent theories using the JKR theory, namely that the effect of adhesion depends critically on the fractal dimension. For $D<2.5$, the case which includes the vast majority of natural surfaces, there is an expected strong effect of adhesion. Only for large fractal dimensions, $D>2.5$, seems for large enough magnifications that a full fractal roughness completely destroys adhesion. These results are partly paradoxical since strong adhesion is not observed in nature except in special cases. A possible way out of the paradox may be that the conclusion is relevant for the near full contact regime, where the strong role of flaws at the interfaces, and of gaps full of contaminant, trapped air or liquid in pressure, needs to be further explored. If conditions near full contact are not achieved on loading, probably the conclusions of classical asperity theories may be confirmed.
\end{abstract}

Keywords:

Roughness, Adhesion, Fuller and Tabor's theory, fractals 


\section{Introduction}

Adhesion between elastic bodies was relatively unexplored until the last few decades, and this is reflected in the very marginal role it has in the otherwise very comprehensive book of K.L. Johnson (1985), despite Johnson himself is one of the authors of one of the most important papers on adhesion (on adhesion of elastic spheres, the JKR theory, Johnson et al., 1971, which has over 5000 citations at present). This is obviously because until sufficiently accurate and high-resolution technique were developed, adhesion was hard to measure, because roughness, it was commonly observed and explained, destroys the otherwise very strong field of attraction between bodies, which should in principle make them stuck to each other at the theoretical strength of the material. JKR theory itself was developed having in mind the special cases where adhesion can indeed be measured at the macroscopic scales, using very soft materials like rubber and gelatin spheres, clean and with extremely smooth surfaces. Today, there is however interest in both scientific and technological areas also at small scale, where very smooth surfaces for example in information storage devices result in adhesive forces playing a more crucial role than in more conventional tribological applications. On the other hand, when people have started to study adhesion in Geckos, which adhere to just about any surface, being it wet or dry, smooth or rough, hard or soft, with a number of additional extraordinary features (self-cleaning, mechanical switching), interest is emerging on how to reproduce these capabilities in "gecko inspired synthetic adhesives". The interest stems on the fact that adhesion cannot be produced on hard rough surfaces, and therefore only the strikingly complex hierarchical structure of the gecko attachment can produce the macroscopic values of load that Gecko can sustain.

The hierarchical structure of the gecko attachment (about three million microscale hairs (setae) which in turn each branch off into several hundreds of nanoscale spatula, totalling perhaps a billion spatula) makes one wonder why

the multiscale nature of surface roughness also could not show an effect of adhesion enhancement. Indeed, at least one model of adhesion of solid bodies (that of Persson and Tosatti, 2001, PT in the following), does show adhesion persistence and even enhancement. There seems to be a qualitative difference for surfaces with fractal dimensions below 2.5, which turns out to be the case in most if not the totality of the commonly observed rough surfaces 
(Persson, 2014). In general, it is hard to measure strong adhesion, despite the van der Waals interactions in principle are orders of magnitude larger than atmospheric pressure - this "adhesion paradox" (Pastewka and Robbins, 2014, Persson et al., 2005) has been linked to surface roughness, but the explanations of the paradox have been different, the latest very interesting one being due to Pastewka and Robbins (2014), which is a very promising parameter-free theory that shows how adhesion changes contact area and when surfaces are sticky — but mostly in a regime near small contact areas. Pastewka and Robbins (2014) conclude that "For most materials, the internal cohesive interactions that determine elastic stiffness are stronger than adhesive interactions, and surfaces will only stick when they are extremely smooth. Tape, geckos, and other adhesives stick because the effect of internal bonds is diminished to make them anomalously compliant". This conclusion seems in qualitative agreement with the classical asperity theory, except that Pastewka and Robbins use in their model quantities related to slopes and not to heigths and therefore are in quantitative disagreement.

Persson (2002a, 2002b) introduced more elaborate version of the theory, which solves the partial contact problem also, and the coupling of the two aspects (effective energy due to roughness in full contact, and its use in a partial contact with a diffusion model) makes the limit behaviour for very short wavelengths difficult to capture, and motivated us to search a possibly simpler, more traditional picture.

The traditional asperity model of Fuller and Tabor (1975), today is not considered to be adequate because of its many assumptions on geometry and absence of interaction, showed that adhesion and pull-off force is reduced very easily at macroscopic scale by roughness. Even extremely tiny amounts of roughness, of the order of the pull-off distance for the highest asperities in contact, make the pull-off force orders of magnitude lower than the nominal value. FT theory seemed to be in good agreement with the experiments, within the limits of their accuracy. The only case where it seemed contradicted by some experimental evidence, was in some measurements of adhesion in highly viscoelastic solids (Fuller and Roberts, 1981, Briggs and Briscoe 1977). These experiments indeed showed an enhancement of adhesion with roughness, which was not expected in the pure elastic FT model. More recent evidence comes from the cleverly designed experiments using a two-scale axisymmetric problem with roughness between gelatin and perspex flat rough plates, by Guduru and his group (Guduru (2007), Guduru et al (2007), Waters et al (2009)). They showed clearly that an elastic JKR 
analysis explains the strong increment of pull-off forces observed (an order of magnitude increase), and that this comes with irreversible energy dissipated in many jumps of the force-area curve.

In this paper, we shall try therefore a new model for a rough surface, completely different from either asperity models, and PT model (or Persson, 2002a, 2002b). The model is based on the very simple idea Johnson used several times in analyzing contacts near full contact, and which in turn could be attributed to Bueckner (1958): namely, that the gaps in an otherwise full contact are cracks that cannot sustain finite stress intensity factors in the case of pure mechanical contact without adhesion, or that can sustain the appropriate stress intensity factor corresponding to the toughness $K_{I c}$ (in terms of surface energy, $G_{c}=K_{I c}^{2} / E$ ), in the case of adhesion. Further, it was used more recently by Xu et al. (2014) (XJM theory) for a random rough surface near full contact but without adhesion, whose model in fact inspired the present extension to the case with adhesion.

\section{Preliminary remarks on a single sinusoid contact}

Before embarking into the full rough surface case, it is crucially important to understand qualitatively the mechanics of adhesion near full contact. The best strategy is to start from the relatively simple behavior of a single sinusoidal contact, as studied quantitatively by Ken Johnson under the JKR regime assumption (Johnson, 1995). Taking therefore a sinusoid (in either 1D or full 2D) with $\lambda$ wavelength, $h$ amplitude, and considering the limit case without adhesion $p^{*}=\pi E \frac{h}{\lambda}$ is the compressive mean pressure to flatten the sinusoid and achieve full contact, the adhesive case follows curves of area-load described in Fig.1, where we have considered the case of a 1D profile for simplicity because it is fully analytical, whereas probably the $2 \mathrm{D}$ case cannot be solved in closed form, except near full contact and near pulloff. Starting from the case of "low adhesion", $\alpha<0.6$, we can describe the behavior during loading as follows. The curve has two extremes, a minimum and a maximum: under zero load, the contact jumps into a state of contact given by the intersection of the curve with the load axis. Upon further increase of the load, it follows the stable curve, until it jumps into full contact at the maximum. At this point the strength is theoretically infinite (more precisely, the theoretical stress of the material, which is very high) unless we postulate the existence of some flaw of trapped air, as Johnson suggests, 
and which gives a bounded tensile pressure for returning on the curve at the maximum. Upon further unloading, the curve is followed stably until the minimum is reached (therefore we have a new part of the curve that is now stable, that under negative loads), where pull-off, or jump out-of-contact, is obtained. For a "critical" value of adhesion, which depends on modulus of the material as well as the two length scales in the problem, the surfaces will spontaneously snap into contact at zero load. This occurs for Johnson's parameter

$$
\alpha=\sqrt{\frac{2 \lambda \Delta \gamma}{\pi^{2} h^{2} E}}>0.6
$$

where $\Delta \gamma$ is the surface energy. What matters in particular to the present investigation is that the original contact curve (that without adhesion) changes sharply shape when adhesion is introduced, since the negative pressure region appears which is crucial to understand pull-off loads, and the transition towards infinite tension is also introduced, rather than having full contact at specific value of (compressive) pressure. However, for what concerns the condition of jump into full contact, this is essentially given by the maximum of the curve which is a perturbation of the contact solution - the curve for negative tension and pull-off requires a detailed analysis of the regime of low contact, and we believe for this part the model of Fuller and Tabor (1975) is a good starting point, as the Hertzian JKR solution is a good starting point to study the sinusoidal indenter. Notice finally that for $\alpha>0.6$ we simply have that the pull-off force will be load dependent.

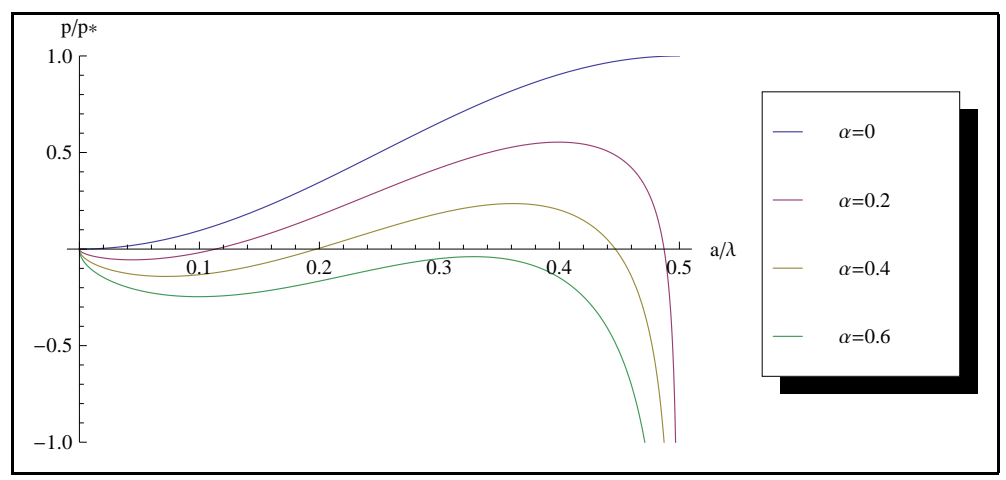

Figure 1. The relationship between $p / p^{*}$ and contact area ratio $a / \lambda$ for Johnson's JKR solution of the single 1D sinusoidal adhesive contact problem. The change from the pure contact case $\alpha=0$, to adhesive case $\alpha=0.2,0.4,0.6$. 


\section{The model}

In the classical random process theory, the pressure to cause full contact, $p_{f c}$, is a random variable, whose variance is easily related in terms of the power spectrum density (PSD) of the profile or of the surface (see Manners and Greenwood, 2006, Persson, 2001, Persson and Tosatti, 2001)

$$
V(\zeta)=\left\langle p_{f c}^{2}\right\rangle=\frac{1}{4} E^{* 2} \sigma_{m}^{2}=\frac{1}{4} E^{* 2} m_{2}
$$

where $\sigma_{m}^{2}=\left\langle\left(\frac{d z}{d x}\right)^{2}\right\rangle=m_{2}$ is the variance of the slopes. $E^{*}$ here is the combined plane strain modulus of the contact materials. The distribution of pressures in full contact is also a Gaussian distribution, namely

$$
P(p, \bar{p}, V)=\frac{1}{\sqrt{2 \pi V}} \exp \left[-\left(\frac{(p-\bar{p})^{2}}{2 V}\right)\right]
$$

This means that, strictly speaking, there is always a tail of negative (tensile) pressures, and indeed Persson's solution for the adhesionless contact problem simply truncates this distribution by subtracting from it a specular distribution, with negative mean pressure, obtaining in fact a Rayleigh distribution.

For typical self-affine surfaces, eliminating the so called "roll-off" wavelength, the power spectrum is a power law above a certain long wavelength cut-off (wavenumber $q_{0}$ )

$$
C(q)=\left\{\begin{array}{cc}
0 & \text { for } q<q_{0} \\
\frac{H}{2 \pi}\left(\frac{h_{0}}{\lambda_{0}}\right)^{2}\left(\frac{q}{q_{0}}\right)^{-2(H+1)} & \text { for } q>q_{0}
\end{array}\right.
$$

For any finite short wavelength cutoff $q_{1}=\zeta q_{0}$, the moments are bounded, but in the limit, $m_{0}$ only is bounded (the variance of the heights), whereas $m_{2}$ diverges as well as the other higher order ones. For this reason, it is already well known that full contact cannot occur for any finite pressure, in the fractal limit, as it is for determinist fractal profiles like those defined with the Weierstrass series in Ciavarella et al. (2000). In the random case, Persson's solution, which is approximate but qualitatively correct (Wolf et al, 2014) shows that the contact area is only complete for infinite applied compression.

According to Bueckner's principle (1958), as used by Johnson (1995) and XJM (Xu et al. 2014), we need to look carefully at the tensile stresses 
of the full contact pressure solution, which are applied by superposition to the gaps area, and compute the stress intensity factors. Considering this contact pressure solution as a random process, it is clear that its moments will correspond to the original surface moments, shifted by a factor 2 and multiplied by $\frac{1}{4} E^{* 2}$

$$
m_{0}^{p}=\left\langle p_{f c}^{2}\right\rangle=\frac{1}{4} E^{* 2} m_{2} ; \quad m_{2}^{p}=\frac{1}{4} E^{* 2} m_{4} ; \quad m_{4}^{p}=\frac{1}{4} E^{* 2} m_{6}
$$

According to Taylor series expansion therefore, as Manners and Greenwood (2006), and XJM (Xu et al. 2014) also suggest, the tensile part of the full contact solution can be approximated with quadratic equations near the pressure tensile summits, similarly to what is done for asperity theories for the real geometry of the surface. This generates a model of isolated "noncontact" areas. The XJM model (Xu et al. 2014) shows that this leads to the following:-

- the individual tensile area is

$$
A_{t}=\pi a_{t}^{2}=2 \pi R_{p}(p-\bar{p})
$$

where $R_{p}$ is the radius of the asperity full contact pressure distribution, and $(p-\bar{p})$ is the pressure on the asperity (the full contact solution in the tensile regions, with a change in sign due to the Bueckner's superposition (1958));

- the non-contact area can be exactly shown, according to Bueckner's principle, to correspond to a pressurized crack.

- For the pure contact case, the stress intensity factor (SIF) has to be zero along the boundary, axysimmetric by assumption (consideration of elliptical form do not change the results significantly)

$$
K_{I}=\frac{2 \sqrt{c_{t}}}{\sqrt{\pi}}(p-\bar{p})\left[1-\frac{2}{3} \frac{c_{t}^{2}}{a_{t}^{2}}\right]=0
$$

leading to the conclusion that the non-contact area is larger than the tensile stress area, and in particular, it is $3 / 2$ in size of the original tensile area,

$$
c_{t} / a_{t}=\sqrt{3 / 2}
$$


Notice however that the condition is also satisfied trivially by the solution $c_{t}=0$ - since clearly the fact that the size of the gap goes to zero is also a solution is problematic for studying that limit. The point is we also have another condition and not just LEFM: namely, that there cannot be any tension, which means actually the strength is zero. If we consider adhesion, there can be tension, up to the theoretical strength.

- For the case where there is in fact surface energy, the condition becomes more elaborate

$$
K_{I}=\frac{2 \sqrt{c_{t}}}{\sqrt{\pi}}(p-\bar{p})\left[1-\frac{2}{3} \frac{c_{t}^{2}}{a_{t}^{2}}\right]=K_{I c}
$$

and for example if $\frac{c_{t}^{2}}{a_{t}^{2}}=1$, we would have an open crack $K_{I}=\frac{2 \sqrt{c_{t}}}{\sqrt{\pi}}(p-\bar{p})>$ 0 that would tend to propagate. We expect naturally $\frac{c_{t}^{2}}{a_{t}^{2}}<3 / 2$ as an effect of adhesion.

- There cannot be solutions below the size where we need to take into account the transition towards a strength criterion - $(p-\bar{p})=\sigma_{t h}$. However, this is of concern only if there is a solution of full contact with finite pressure, and in any case, the suggestion of Johnson to consider at this point the presence of trapped air and we shall return later on this point.

Substituting (6), into (8), we have an equation for $c_{t}$

$$
\frac{2 \sqrt{c_{t}}}{\sqrt{\pi}}(p-\bar{p})\left[1-\frac{2}{3} \frac{c_{t}^{2}}{2 R_{p}(p-\bar{p})}\right]=K_{I c}
$$

It is clear that we cannot solve this equation easily in a rigorous sense, since $(p-\bar{p})$ is a random variable. This equation (10) is an implicit equation which defines

$$
c_{t}=g\left(p-\bar{p}, R_{p}, K_{I c}\right)
$$

and acts like the basic function defining the local area as a function of the "separation" $p-\bar{p}$ in the equivalent asperity model created by the tensile full contact pressure "surface". 


\subsection{The adhesion-less case}

It is useful to derive separately this case, as done by $\mathrm{Xu}$ et al (2014). From (10), with $K_{I c}=0$

$$
c_{t}^{2}=3 R_{p}(p-\bar{p})
$$

There is no minimum tensile tension that can be sustained (unlike with adhesion) and the integration proceeds simply as suggested by eqt.38 of $\mathrm{Xu}$ et al (2014)

$$
\left(\frac{A_{n c}(\bar{p})}{A_{0}}\right)=3 \pi R_{p} \eta \int_{\bar{p}}^{\infty}(p-\bar{p}) \Phi(p) d p
$$

where $\eta$ is the asperity density of the full contact pressure surface, $\Phi(p)$ is the distribution of the pressures summits in this surface, and hence it can be solved easily. Notice the integral is simply of the same mathematical form as in the standard Greenwood and Williamson's (1966) theory, where mean separation is replaced by mean pressure, and the geometrical surface is replaced by the pressure surface. In the present form, we obtain

$$
\frac{A_{n c}(\bar{p})}{A_{0}}=3 \pi R_{p} \eta \sqrt{V}\left(\frac{1}{\sqrt{2 \pi}} \exp \left[-\left(\frac{\bar{p}^{2}}{2 V}\right)\right]-\frac{1}{2} \frac{\bar{p}}{\sqrt{V}} \operatorname{Erfc}\left(\frac{\bar{p}}{\sqrt{2 V}}\right)\right)
$$

Further, at sufficiently large magnifications, $\sqrt{V} \sim \zeta^{1-H}$, and $R_{p} \simeq$ $\frac{0.375}{E^{*}} \sqrt{2 \pi / m_{6}} \sim \zeta^{-(3-H)}$, while also the density of asperities $\eta=\frac{m_{6}}{6 \sqrt{\pi} m_{4}} \sim$ $\frac{\zeta^{(6-2 H)}}{\zeta^{(4-2 H)}}=\zeta^{2}$, which gives $R_{p} \eta \sqrt{V} \rightarrow \zeta^{-(3-H)} \zeta^{2} \zeta^{1-H}=\delta$ where $\delta$ is a prefactor of the order 1 (the exact prefactor to make the area of contact zero at zero pressure is $\delta=\sqrt{2 \pi} \sim 2.5$, but this is not the correct value at large pressures where we are concentrating our efforts)

$$
\frac{A_{n c}(\bar{p})}{\delta A_{0}} \rightarrow \frac{1}{\sqrt{2 \pi}} \exp \left[-\left(\frac{\bar{p}^{2}}{2 V}\right)\right]-\frac{1}{2} \frac{\bar{p}}{\sqrt{V}} \operatorname{Erfc}\left(\frac{\bar{p}}{\sqrt{2 V}}\right)
$$

This suggests that if we want to keep a constant value of given area of gap, upon increasing magnification, we need to keep $\frac{\bar{p}}{\sqrt{2 V}}$ constant, i.e. increase the mean pressure without limit. This is the well know behavior of pure contact problem, and it is confirmed here.

\subsection{Energy balance equation at boundary of gaps}

It is important to discuss in details equation (10), as it governs the basic behavior of the gaps during the loading process. First of all, it is easier to 
manipulate it in terms of the tension on the "pressurized cracks", as (notice that $R_{p}$ being the radius of the pressure surface, has dimensions $m^{4} / N$ ), by rewriting it as

$$
(p-\bar{p})=\frac{2}{3} \frac{c_{t}^{2}}{2 R_{p}}+\frac{\sqrt{\pi}}{2 \sqrt{c_{t}}} K_{I c}
$$

where the second term cancels out obviously in the adhesion-less case, but also with adhesion at very large $c_{t}$. A few curves could be plotted to show the general trend of introducing a minimum which clearly corresponds to the maximum in the case in Johnson's sinusoidal case (see Fig.1) near the (unstable) transition to full contact. We prefer however to arrive at a cleaner plot, which will be in Fig.2, to include $K_{I c}$ in a unique curve. Notice that as we increase the mean compression in the contact, the actual value of tension in the gaps decreases - therefore, the loading progresses here by reducing the pressure on the y-axis. The minimum occurs at $\frac{\partial}{\partial c_{t}}(p-\bar{p})=$ $\frac{2}{3} \frac{c_{t}}{R_{p}}-1 / 2 \frac{\sqrt{\pi}}{2 c_{t}^{3 / 2}} K_{I c}=0$, which gives

$$
c_{t \min }=\left(\frac{3}{8} \sqrt{\pi}\right)^{2 / 5}\left(R_{p} K_{I c}\right)^{2 / 5}
$$

in which case, for $\psi=\left(\frac{1}{3}\left(\frac{3}{8} \sqrt{\pi}\right)^{4 / 5}+\frac{\sqrt{\pi}}{2\left(\frac{3}{8} \sqrt{\pi}\right)^{1 / 5}}\right)=1.2021$

$$
(p-\bar{p})_{\min }=p_{0}=\psi\left(\frac{K_{I c}^{4}}{R_{p}}\right)^{1 / 5}
$$

where $R_{p}$ is a radius of pressure "asperity". Hence, we can rewrite (16) as

$$
\frac{(p-\bar{p})}{p_{0}}=\frac{2}{3} \frac{1}{\psi} \frac{c_{t}^{2}}{2 R_{p}}\left(\frac{R_{p}}{K_{I c}^{4}}\right)^{1 / 5}+\frac{1}{\psi} \frac{\sqrt{\pi}}{2 \sqrt{c_{t}}} K_{I c}\left(\frac{R_{p}}{K_{I c}^{4}}\right)^{1 / 5} .
$$

The result comes clean defining

$$
\widehat{p}=\frac{(p-\bar{p})}{p_{0}} \text { and } \widehat{c}_{t}=\frac{c_{t}}{c_{t \min }}
$$

Hence, we can rewrite (19) as

$$
\widehat{p}=\frac{1}{5}\left(\widehat{c}_{t}^{2}+\frac{4}{\sqrt{\widehat{c}_{t}}}\right)
$$


and this is indeed the curve plotted in Fig. 2. In the adhesion-less case, $(p-\bar{p})_{\min } \rightarrow \infty$, and $c_{t \min } \rightarrow \infty$ and hence the curve diverges to infinity and hence this simple unique curve for the adhesive case represents a discontinuity and the dimensional quantities should be plotted to see more clearly the transition from the adhesion-less case to the adhesive one.

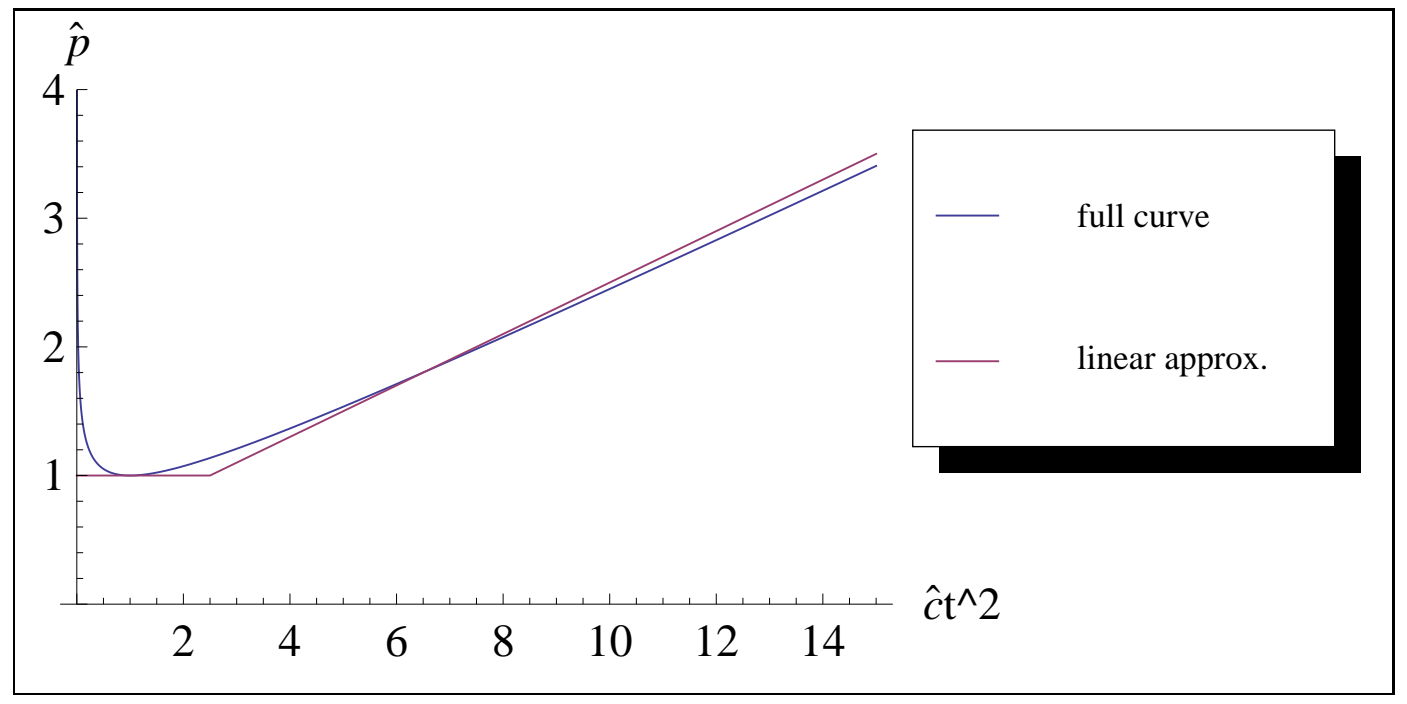

Figure 2. The relationship between peak dimensionless tension $\widehat{p}$ applied on a pressurized isolated gap and the size of the dimensionless gap radius squared $\widehat{c}_{t}^{2}$, together with a linear asymptotic approximation which turns out to be reasonably accurate also at low tensions. The minimum in the curve, which leads to the transition to full contact, is obviously overestimated by the approximate curve, so there will be a minor spurious effect of increase of area of the gaps. The way the plot is constructed doesn't permit to see the limit of pure contact (adhesive-less case), since both $c_{t \min }$ and $p_{0}$ are zero in that limit, and hence the adhesive-less curve becomes the very tail of the present one at infinity

Further, considering that we want to make estimates of the area of the gap that remains in contact, it is clear that a very good approximate solution could be

$$
\widehat{p}=\left\{\begin{array}{cc}
\frac{1}{5} \widehat{c}_{t}^{2}+\frac{1}{2} & \text { for } \widehat{c}_{t}^{2}>\frac{5}{2} \\
1 & \text { for } 0<\widehat{c}_{t}^{2}<\frac{5}{2}
\end{array}\right.
$$


since the other branch of the equation is unstable. The only significant approximation this introduces, it is to overestimate the size of the gap region radius just before jumping into contact. However, while this will only change some prefactors by small amounts, it simplifies the study of the problem enormously.

Now, supposing we start from a contact which doesn't jump into contact completely and with an applied compressive load - but that gaps area do exist. It is clear that the (22) solution holds for each gap, depending on the local tension arising from cancelling the tension in the full contact pressure, and at any given mean applied load, each gap will have a certain level of pressure and therefore a certain equilibrium size as per (22), which may include some gaps closing. We need an integration process to establish the total area of gaps, and therefore the complementary remaining contact area. Also, upon loading, the mean compressive pressure increases, and hence the tensile pressure on each gap decreases, so that more gaps will tend to reach the condition $\widehat{p}=1$. Therefore, a certain number of gaps will close, and the others will reduce their size. To understand if the final state is full contact or not, we should consider the adhesion-less case for reference. In this case, approximately, Persson's solution indicates that only $\sqrt{V}$ is the parameter ruling the contact area size. If we increase the short wavelength content, increasing $\sqrt{V}$, for a given contact area, we have to increase the mean pressure in proportion. Given $\sqrt{V}$ grows unbounded, the pressure to obtain any value of contact area (in fact, not just full contact), grows unbounded.

Repeating this reasoning here, we need to observe if, for a given condition with adhesion, the contact area depends only on the ratio $p / \sqrt{V}$ or not.

Rewriting and inverting the equation (22) in terms of the gap radiuses

$$
\widehat{c}_{t}^{2}=\left\{\begin{array}{cc}
5\left(\widehat{p}-\frac{1}{2}\right) & \text { for } \widehat{p}>1 \\
0 & \text { for } \widehat{p}<1
\end{array}\right.
$$

and in dimensional terms

$$
c_{t}^{2}=\left(\frac{3}{8} \sqrt{\pi}\right)^{4 / 5}\left(R_{p} K_{I c}\right)^{4 / 5}\left\{\begin{array}{cl}
5\left(\frac{(p-\bar{p})}{p_{0}}-\frac{1}{2}\right) & \text { for } \frac{(p-\bar{p})}{p_{0}}>1 \\
0 & \text { for } \frac{(p-\bar{p})}{p_{0}}<1
\end{array}\right.
$$

or

$$
\pi c_{t}^{2}=3 \pi R_{p}\left[(p-\bar{p})-p_{0}\right], \text { for }(p-\bar{p})>p_{0}
$$




\subsection{Integration and results}

We shall neglect the variation of $R_{p}$ with height, as otherwise the integration becomes too cumbersome. We simply assume a mean value given by random process theory, and develop an integration of the type

$$
\frac{A_{n c}(\bar{p})}{A_{0}}=\eta \int_{\bar{p}+p_{0}}^{\infty} \pi c_{t}^{2} \Phi(p) d p
$$

where the function for $c_{t}$ is now given in (24), which results in

$$
\frac{A_{n c}(\bar{p})}{A_{0}}=3 \pi R_{p} \eta \sqrt{V}\left[\frac{1}{\sqrt{2 \pi}} \exp \left[-\left(\frac{\left(\bar{p}+p_{0}\right)^{2}}{2 V}\right)\right]-\frac{\bar{p}+p_{0}}{2 \sqrt{V}} \operatorname{Erfc}\left(\frac{\bar{p}+p_{0}}{\sqrt{2 V}}\right)\right]
$$

which agrees with the adhesiveless case when $p_{0}=0$. In this format, it is clear that the effect of $p_{0}$ is exactly analogous to an increase of the mean pressure. The non-contact area would tend to stay constant with magnification $R_{p} \eta \sqrt{V} \rightarrow \delta$ now if we keep increase, instead of the applied pressure proportionally to $\sqrt{V} \sim \zeta^{1-H}$, only $p_{0}$, and this is simple to study. At sufficiently large magnifications, looking at the parameter (18), and considering the usual scaling arguments on the PSD and its moments

$$
\frac{p_{0}}{\sqrt{V}}=\frac{\psi}{\sqrt{V}}\left(\frac{K_{I c}^{4}}{R_{p}}\right)^{1 / 5} \sim \frac{m_{6}^{1 / 10}}{m_{2}^{1 / 2}} \sim \frac{\left(\zeta^{(6-2 H)}\right)^{1 / 10}}{\zeta^{1-H}} \sim \zeta^{\frac{2}{5}(2 H-1)}
$$

where the exponent $\theta=\frac{2}{5}(2 H-1)$ is positive for $H>0.5$ (low fractal dimensions), when the dimensionless $\frac{p_{0}}{\sqrt{V}}$ increases with magnification, or negative otherwise.

Hence, for low fractal dimensions, and a given applied mean pressure (including zero), the non-contact area tends to decrease without limit, implying the tendency to full contact. Naturally, the tendency will be stronger the higher $H>0.5$ i.e. the farther from the limit case of $H=0.5$. There seems to be some connection to the conclusions and the parameters involved in the "effective adhesion energy" in Persson and Tosatti (2001), which leads them to suggest that adhesion persists for low fractal dimensions $D<2.5$ (the real range of surfaces, see Persson (2014)). In their theory, they obtain from the elastic energy associated to the deformation in balance with surface energy of a full spectrum of frequencies, a parameter which seems related to

$$
E m_{1}=\frac{E}{4} h_{0} q_{0} f(H) \sim \zeta^{1-2 H}
$$


where $m_{1}$ is the first order moment of the PSD. This has the same qualitative power law behaviour (besides the $\frac{2}{5}$ factor), but it should be emphasized different from - in PT coming from the first moment of the surface, and in our case from a combination of roots of the second and 6-th moment.

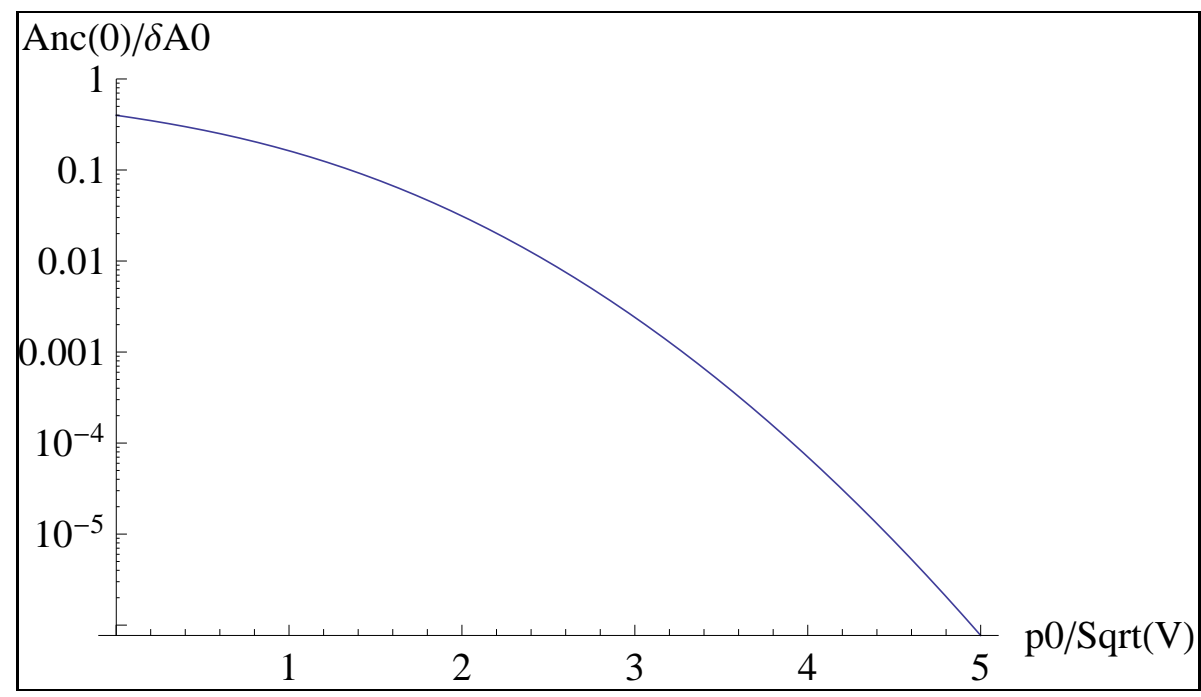

Figure 3. The area of gaps $\frac{A_{n c}(0)}{A_{0}}$ for zero applied pressure decreases rapidly with the dimensionless adhesion pressure term $\frac{p_{0}}{\sqrt{V}}$ and when $\frac{p_{0}}{\sqrt{V}} \approx 5$ we can assume full contact holds.

Fig. 3 shows a plot of the non contact area at zero applied mean pressure, $\frac{A_{n c}(\bar{p}=0)}{A_{0}}$, which decays very rapidly with the dimensionless ratio $\frac{p_{0}}{\sqrt{V}}$, indicating there is chance of spontaneous full contact for $H>0.5$, although the model is clearly approximate in that range since we are far from full contact.

For $H<0.5$ and hence large fractal dimensions, the dimensionless ratio $\frac{p_{0}}{\sqrt{V}}$ will decrease with magnification, and hence we return at large magnifications to the case of pure adhesion-less contact, for which we expect at zero load simply zero area.

The situation is clear therefore also with applied compressive forces to the contact. Contrary to the case without adhesion, where no mean pressure is sufficient to squeeze the contact flat, here for large Hurst exponent $H>0.5$, the non-contact area decreases asymptotically to zero and the trends of the zero applied pressure are confirmed, as clearly seen in Fig.4, where we assume the power law scaling $\frac{p_{0}}{\sqrt{V}}=\zeta^{\frac{2}{5}(2 H-1)}$ with $\frac{p_{0}}{\sqrt{V}}(\zeta=1)=1$. 

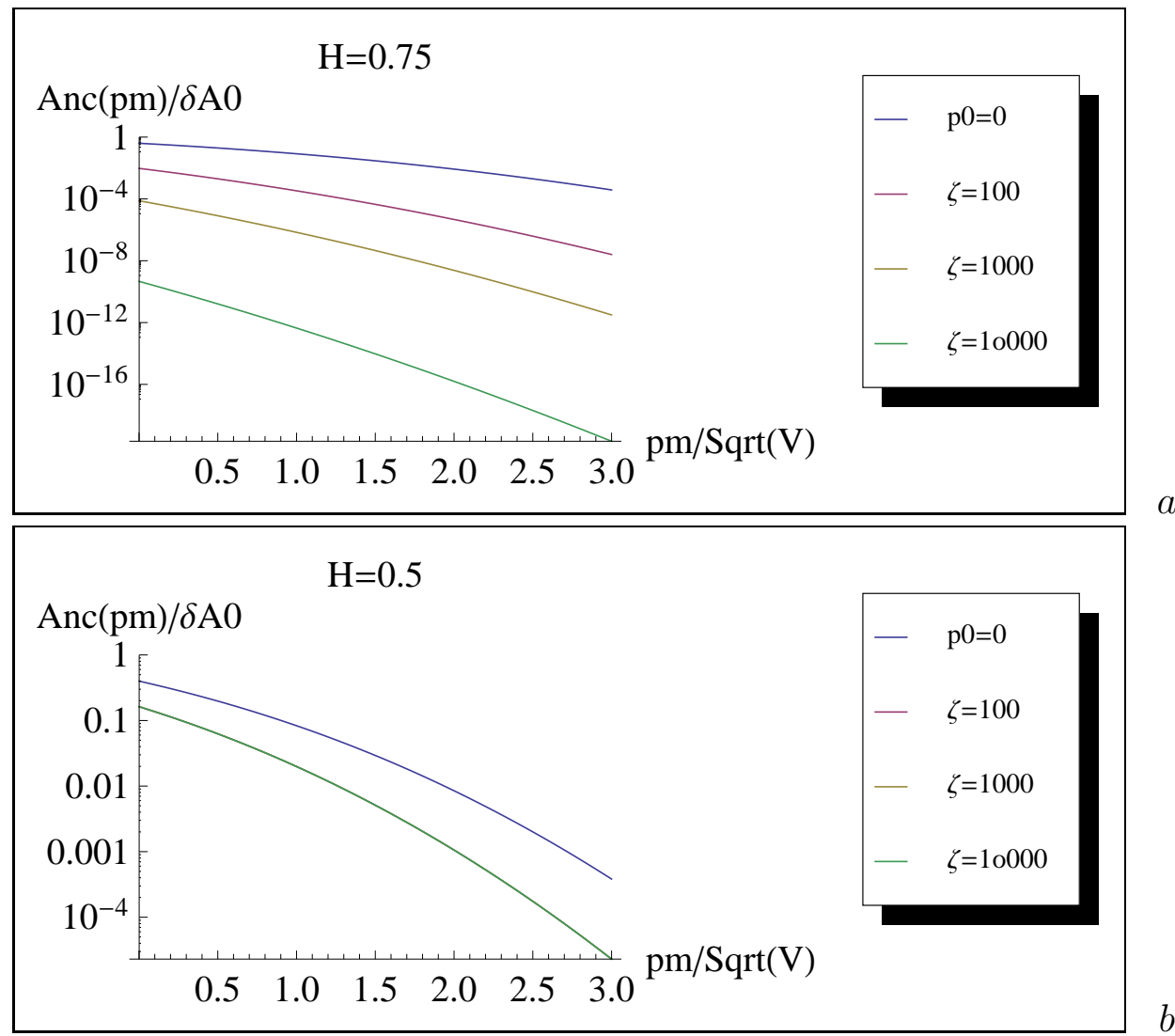

$b$

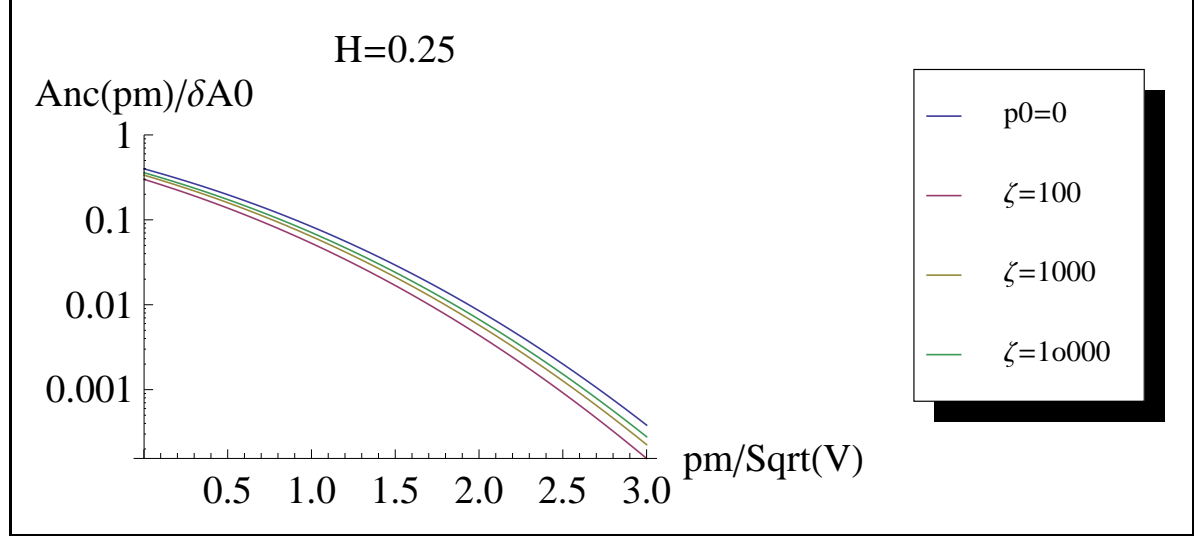

c

Figure 4. The area of gaps (complementary to the real contact area) for different $H=0.75,0.5,0.25$ as a function of the dimensionless applied compression $\frac{\bar{p}}{\sqrt{V}}$ shows that the contact becomes practically full at finite values of the $\frac{\bar{p}}{\sqrt{V}}$ contrary to the 
purely mechanical case for $H>0.5$. Vice versa, for $H=0.5$, this tendency is very similar to the adhesive-less contact and for $H=0.25$ there is no significant difference with the adhesive-less case. We assume power law scaling for $\frac{p_{0}}{\sqrt{V}}$ with $\frac{p_{0}}{\sqrt{V}}(\zeta=1)=1$.

\section{An alternative derivation}

We obtained a GW-like asperity full contact pressure surface model for adhesion and more in general, in the adhesive-less version of this (obtained putting $\left.p_{0}=0\right)$, $\mathrm{Xu}$ et al (2014) noticed the close connection to the results with the Persson (2001) theory of contact. Xu et al (2014) introduced varius advanced asperity models. In particular, the most advanced theories which recognize the variation of mean radius with heigth, and for large separations they are considered to be exact - large separation being here, in the equivalent pressure "surface" model, large pressures. A further attempt is of interest, since it brings very close comparison with Persson's theory. As recognized by the advanced theories of BGT or Carbone (2009), the contact area can be connected the bearing area ratio $A_{b}(t) / A_{0}$ at large separations to the contact area, and hence, here a given separation $d$, it depends only on a single parameter of the random process surface $\left(m_{0}\right.$ is the zero-th order moment of the spectral density of the surface):

$$
\frac{A_{b}(t)}{A_{0}}=\frac{1}{2} \operatorname{Erfc}\left(\frac{t}{\sqrt{2}}\right)
$$

where separation is made non-dimensional by the ratio $t=d / m_{0}^{1 / 2}$. When translating this into the "pressure surface" model, this should give an area of "non-contact" as a function of mean pressure $p$, made non-dimensional by dividing it by the $0-t h$ order moment of the pressures

$$
m_{0}^{p}=\left\langle p_{f c}^{2}\right\rangle=V
$$

defined above (2), which in fact translates onto $\frac{1}{4} E^{*} m_{2}$. Here, we recognize already some features of the Persson's equation, contrary to the more "accurate" asperity models in $\mathrm{Xu}$ et al. (2014) which additionally depend on bandwidth parameter.

Now, in the limit when the mean pressure is zero, we know that by definition, the non-contact area should be 1 , so that the real contact area is zero. 
Therefore, in order for the non-contact area to be 1 at zero separation, we need it to be double of the bearing area (30) at zero separation. Hence, we multiply (30) by factor 2 , and use (31), to get

$$
\frac{A_{n c}(p)}{A_{0}}=\operatorname{Erfc}\left(\frac{p}{\sqrt{2 m_{0}^{p}}}\right)=\operatorname{Erfc}\left(\frac{\sqrt{2} p}{E^{*} \sqrt{V}}\right)
$$

Writing in terms of the complementary term, the area of contact, we get

$$
\frac{A_{c}(p)}{A_{0}}=\frac{1-A_{n c}(p)}{A_{0}}=1-\operatorname{Erfc}\left(\frac{\sqrt{2} p}{E^{*} \sqrt{V}}\right)=\operatorname{erf}\left(\frac{\sqrt{2} p}{E^{*} \sqrt{V}}\right)
$$

Remarkably, this is exactly Persson's equation, in the entire range of pressures, including the low pressure end. Therefore, the use of the bearing area assumption is not limited by extremely large separations in the asymptotic version of the area-separation relationship in BGT's model.

Turning back on the adhesion case, we reached the conclusion that the integration for a GW-equivalent model was of the type

$$
\frac{A_{n c}(\bar{p})}{A_{0}}=3 \pi R_{p} \eta \int_{\bar{p}+p_{0}}^{\infty}\left[p-\left(\bar{p}+p_{0}\right)\right] \Phi(p) d p
$$

and hence, this is exactly equivalent to an adhesiveless contact problem where the mean pressure has been replaced by the sum $\left(\bar{p}+p_{0}\right)$.

Hence, in order to obtain a result consistent to adhesionless Persson's theory in the adhesive case, we need to multiply by a factor $4 / 3$, resulting in

$$
\frac{A_{n c}(\bar{p})}{A_{0}}=\operatorname{Erfc}\left(\frac{\sqrt{2}\left(\bar{p}+p_{0}\right)}{E^{*} \sqrt{V}}\right)
$$

resulting in

$$
\frac{A_{c}(p)}{A_{0}}=\frac{1-A_{n c}(p)}{A_{0}}=1-\operatorname{Erfc}\left(\frac{\sqrt{2}\left(\bar{p}+p_{0}\right)}{E^{*} \sqrt{V}}\right)=\operatorname{erf}\left(\frac{\sqrt{2}\left(\bar{p}+p_{0}\right)}{E^{*} \sqrt{V}}\right)
$$

Comparison with the previous theory shows that the results are qualitatively similar. However, it is easier to plot them in terms of actual contact area, rather than non-contact, because we forces the prefactors to be such, as in the original Persson's theory, to produce zero contact area at zero load, 
in the absence of adhesion. Fig.5 therefore plots eve the range of negative applied load, where in some case the contact is nearly full and hence the assumptions made of isolated gaps in the present model may be fulfilled. The results loose sense if the contact area is small, viceversa.
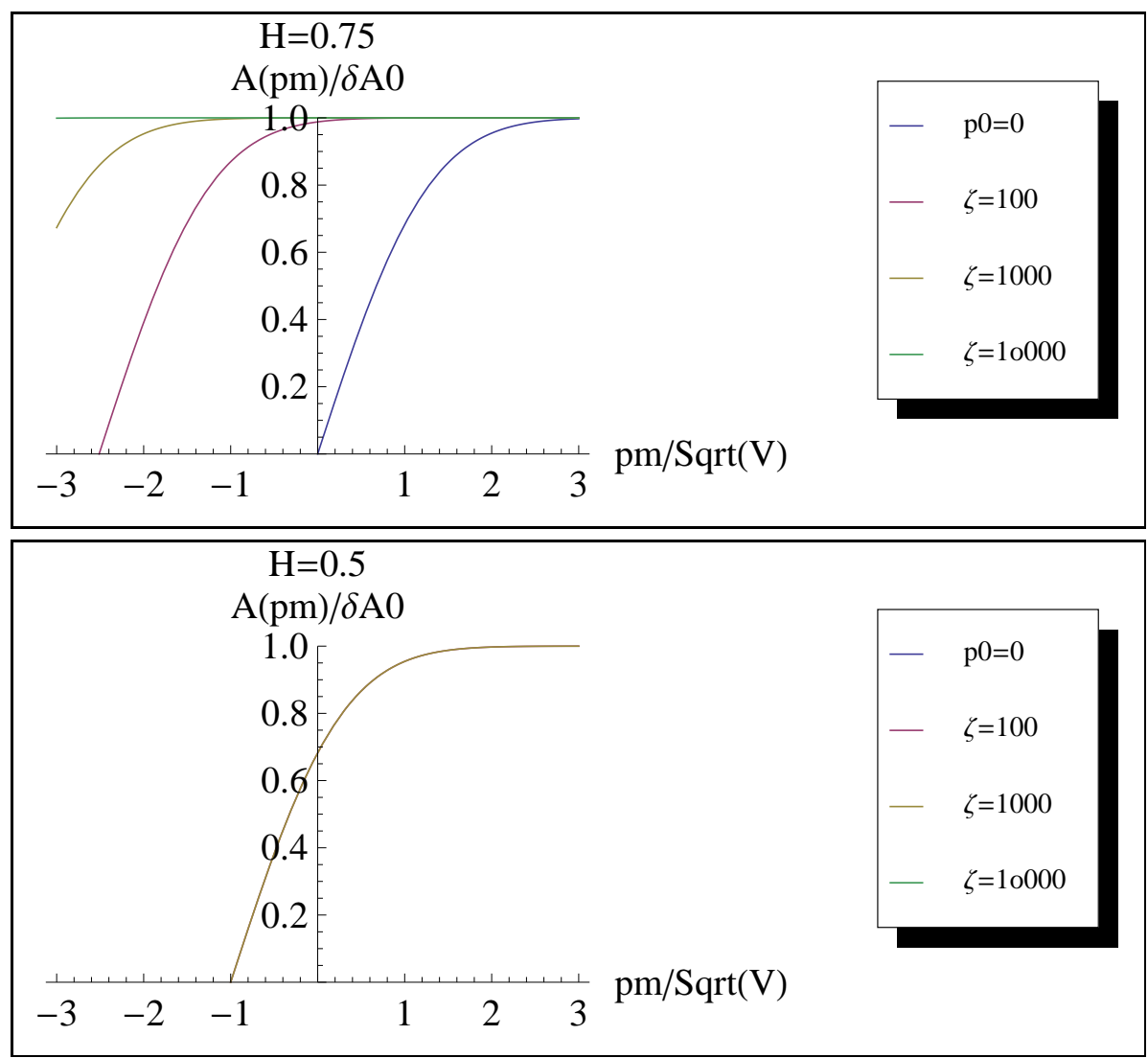

$a$

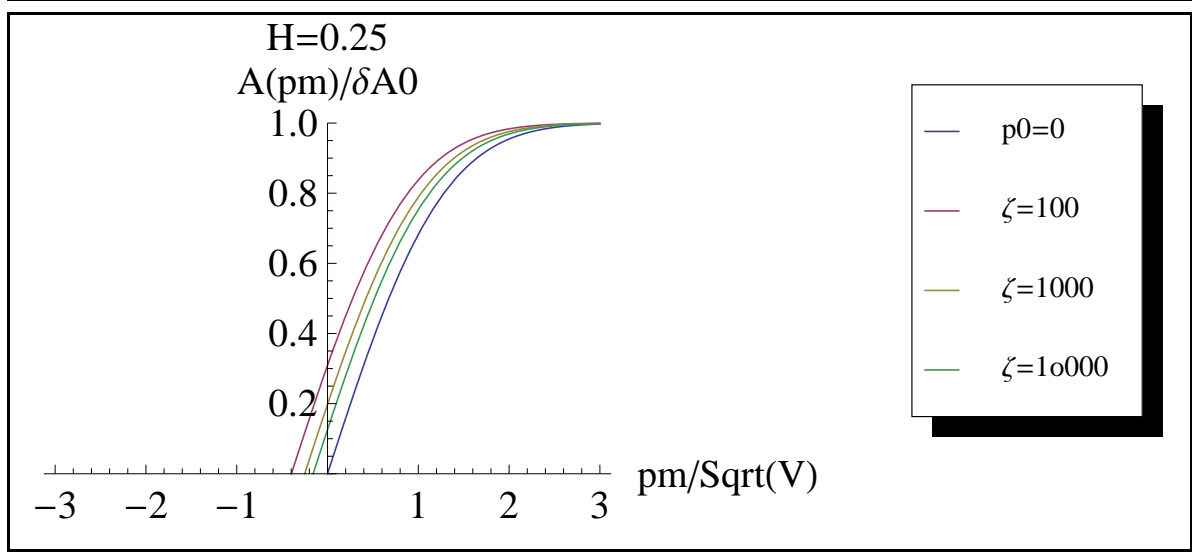

$b$ 
Figure 5. The real contact area for different $H=0.75,0.5,0.25$ as a function of the dimensionless applied compression $\frac{\bar{p}}{\sqrt{V}}$ shows that the contact becomes practically full at finite values of the $\frac{\bar{p}}{\sqrt{V}}$ (and even zero load for $\mathbf{H}=\mathbf{0 . 7 5}$, within the limits of our model). For $H=0.5$, the magnification effect is neutral, whereas for $H=0.25$ there is no significant difference with the adhesive-less

case, and the curves are close to the adhesive-less case. We assume power law scaling for $\frac{p_{0}}{\sqrt{V}}$ with $\frac{p_{0}}{\sqrt{V}}(\zeta=1)=1$.

\subsection{Pull off?}

Let us now discuss the case of pull-off in qualitative terms. This may occur in a range with small contact area, if either the contact doesn't proceed spontaneously into large fractions of the nominal contact area, or if the applied loading doesn't push toward this range. In that case contact is so clearly isolated on asperities, than theories like Fuller and Tabor (1975) would be approximately true.

The problem and limit of the present theory is, insteed, that it cannot deal with the low contact areas, where gaps are interacting and certainly the bends in the area-load curves which would appear in a set of isolated asperity theories are not reproduced here.

The only estimate we can make is therefore to extrapolate pull-off from the point where the contact area is expected to be zero, and this gives.

$$
\frac{A_{c}(p)}{A_{0}}=\operatorname{erf}\left(\frac{\sqrt{2}\left(\bar{p}+p_{0}\right)}{E^{*} \sqrt{V}}\right)=\frac{2 \sqrt{2}}{\sqrt{\pi}} \frac{\left(\bar{p}+p_{0}\right)}{E^{*} \sqrt{V}}=0
$$

which leads to a new meaning for the pressure $p_{0}$

$$
\left(\frac{\bar{p}}{\sqrt{V}}\right)_{\text {pulloff }}=-\frac{p_{0}}{\sqrt{V}}=-\psi\left(\frac{E^{3} G_{c}^{2}}{0.375 \sqrt{2 \pi}}\right)^{1 / 5} \frac{m_{6}^{1 / 10}}{m_{2}^{1 / 2}} \sim-\zeta^{\frac{2}{5}(2 H-1)}
$$

which always increases with magnification. Notice however that in absolute terms, $(\bar{p})_{\text {pulloff }}$ is always increasing for all parameters

$$
\bar{p}_{\text {pulloff }}=-p_{0} \sim-m_{6}^{1 / 10} \sim-\zeta^{\frac{3-H}{5}}
$$

and this result is not easy to beleive, and in opposite constrast to asperity theories but even Pastewka and Robbins (2014) who find stick surfaces only those that are smooth enough (in terms of surface slopes) to have the cohesive energy in the bulk giving up against the adhesion forces at the interface. 


\section{Conclusion}

A new model of adhesion has been discussed and shown to lead to very simple and clear results: there cannot be spontaneous jump into contact for any surface having sufficiently multiscale content, no matter its fractal dimension. The model is devised near the full contact regime, so that the contact consists of a set of isolated gaps whose surfaces are then loaded by Bueckner's principle by the tensile pressures of the "linear" full contact solution, which are approximated by parabola since, by Taylor's expansion, they must have this form near full contact when gaps are closing, and it is easy to write this for a Gaussian surface. The stable branch of the curve of the gap radius vs applied pressure in the gaps are then found imposing the stress intensity factor to be constant along the edge, and a very good approximation turns out to be a linear law with an offset, which permits extremely simple integration, which resemble those of asperity models, and indeed can be considered as a "pressure asperity" model for the pressurized gaps. In the case of no adhesion, as already noticed by Xu et al (2014), the result turn out to be extremely similar to those of Persson's contact theory (2001) which is a widely recognized as a good approximate solution near full contact, and which gives us confidence the results are also very similarly accurate where it tends to be exact in the limit of full contact (for infinite mean pressure applied).

It is shown that a dimensionless ratio governs the contact and a pressure $p_{0}$ can be defined which is scale dependent and includes the energy of adhesion. This pressure has a role equivalent to the mean applied pressure in the equation of the non-contact area and hence since it grows without limit for low fractal dimensions, permits full contact to be achieved for those surfaces. The conclusions cannot be complete of unloading and pull-off force, which require further investigation.

An equation which looks like an extension of Persson's theory for contact mechanics has been derived.

\section{References}

Briggs G A D and Briscoe B J 1977 The effect of surface topography on the adhesion of elastic solids J. Phys. D: Appl. Phys. 10 2453-2466

Bueckner, H., 1958, The propagation of cracks and the energy of elastic deformation. Trans of the Amer Soc of Mech Eng, 80, 1225-1230. 
Ciavarella, M., et al. "Linear elastic contact of the Weierstrass profile." Proceedings of the Royal Society of London. Series A: Mathematical, Physical and Engineering Sciences 456.1994 (2000): 387-405.

Derjaguin, BV and Muller, VM and Toporov, Y.P., 1975, Effect of contact deformations on the adhesion of particles, Journal of Colloid and Interface Science, 53(2), pp. 314-326.

Fuller, K.N.G. , Roberts A.D. 1981. Rubber rolling on rough surfaces J. Phys. D Appl. Phys., 14, pp. 221-239

Greenwood, J.A., Williamson, J.B.P., 1966. Contact of nominally flat surfaces. Proc. R. Soc. London A295, 300-319.

Guduru, P.R. 2007. Detachment of a rigid solid from an elastic wavy surface: theory J. Mech. Phys. Solids, 55, 473-488

Guduru, P.R. , Bull, C. 2007. Detachment of a rigid solid from an elastic wavy surface: experiments J. Mech. Phys. Solids, 55, 473-488

Johnson, K. L., K. Kendall, and A. D. Roberts. 1971. Surface energy and the contact of elastic solids. Proc Royal Soc London A: 324. 1558.

Johnson, K.L., 1985. Contact Mechanics. Cambridge University Press.

Johnson K.L. 1995. The adhesion of two elastic bodies with slightly wavy surfaces, Int J Solids and Struct 32 (3-4), , pp. 423-430

Kellar. A. 2007. Gecko adhesion: structure, function, and applications. MRS bulletin 32.06 (2007): 473-478.

Longuet-Higgins, MS. 1962. The statistical geometry of random surfaces, Proc. Symp. Appl. Math.

Manners, W., Greenwood, J.A., 2006. Some observations on Persson's diffusion theory of elastic contact. Wear 261, 600-610.

Muller, VM and Derjaguin, BV and Toporov, Y.P., 1983, On two methods of calculation of the force of sticking of an elastic sphere to a rigid plane, Colloids and Surfaces, 7(3), pp. 251-259.

Pastewka, L., Robbins MO, 2014. Contact between rough surfaces and a criterion for macroscopic adhesion, Proc Natl Acad Sci USA. 111(9): 32983303.

Persson, B.N.J., 2001. Theory of rubber friction and contact mechanics. J. Chem. Phys. 115, 3840-3861.

Persson, B. N. J., Tosatti. E. 2001. he effect of surface roughness on the adhesion of elastic solids. The Journal of Chemical Physics 115.12: 55975610 . 
Persson B N J 2002 Adhesion between an elastic body and a randomly rough hard surface Eur. Phys. J. E 8 385;

Persson, B. N. J. 2002. Adhesion between elastic bodies with randomly rough surfaces." Physical review letters 89.24: 245502.

Persson, B N J, Albohr, O, Tartaglino, U, Volokitin A I and Tosatti. E 2005. On the nature of surface roughness with application to contact mechanics, sealing, rubber friction and adhesion J. Phys.: Condens. Matter 17 R1, http://arxiv.org/pdf/cond-mat/0502419.pdf)

Persson, B. N. J.. 2014. On the fractal dimension of rough surfaces. Tribology Letters 54.1, 99-106.

Xu, Y., Jackson, R.L., Marghitu, D.B., 2014. Statistical model of nearly complete elastic rough surface contact. Int. J. Solids Struct. 51, 1075-1088.

Yastrebov, V.A., Anciaux, G., Molinari, J.F., 2014. From infinitesimal to full contact between rough surfaces: evolution of the contact area. Int. J. Solids Struct. Available from: <http://arxiv.org/abs/1401.3800>.

Waters, J.F. Leeb, S. Guduru, P.R. 2009. Mechanics of axisymmetric wavy surface adhesion: JKR-DMT transition solution, Int J of Solids and Struct 46 5, 1033-1042

Wolf B.D, Prodanov, N, and Müser, MH. "Systematic analysis of Persson's contact mechanics theory of randomly rough elastic surfaces." Journal of Physics: Condensed Matter 26.35 (2014): 355002. 